\title{
Transesophageal echocardiography in the manage- ment of left atrio-femoral bypass during thora- coabdominal aortic aneurysm repair: a case report
}

\author{
[L'échocardiographie transesophagienne dans la conduite du pontage auriculo-fémoral pendant \\ la réparation d'un anévrysme de l'aorte thoraco-abdominale : une observation]
}

Ashraf Fayad MD FCARCSI, Corey Sawchuk MD FRCPC, Homer Yang MD FRCPC, Claudio Cina MD FRCSC

Purpose: To describe the utility of transesophageal echocardiography (TEE) in a patient undergoing thoracoabdominal aneurysm (TAA) surgery using left atrio-femoral bypass (LAFB).

Clinical features: A 57-yr-old female patient underwent repair of type II TAA. As per institutional routine, LAFB technique was used. Initial difficulty with the pump flow was encountered. TEE images showed that the left atrial cannula was positioned against the left atrial wall. The cannula position was adjusted and the pump flow was established. During different stages of the surgery, TEE was used to monitor the left ventricular cavity size and its function. This allowed the adjustment of LAFB pump flow relative to left ventricular filling and optimal fluid resuscitation in order to maintain both upper and lower body perfusion.

Conclusion: In this patient, TEE was useful to confirm the correct position of the left atrial cannula and for hemodynamic management during LAFB.

Objectif : Décrire l'utilité de l'échocardiographie transœsophagienne (ETO) chez une femme subissant la réparation d'un anévrysme thoraco-abdominal (ATA) par pontage auriculo-fémoral gauche (PAFG).

Éléments cliniques: Une femme de 57 ans devait subir la réparation d'un ATA de type II. Suivant la pratique courante de l'institution, une technique de PAFG a été utilisée. Une première difficulté s'est présentée avec le débit de la pompe. Les images de l'ETO ont montré que la canule auriculaire gauche était placée sur la paroi auriculaire gauche. La canule a été replacée et le débit de la pompe a été établi. À différentes étapes de l'intervention, l'ETO a été utilisée pour surveiller la taille de la cavité ventriculaire gauche et sa fonction. Cet exa- men a permis l'ajustement du débit de la pompe de PAFG relativement au remplissage du ventricule gauche et au remplacement de liquide optimal afin de maintenir la perfusion du tronc supérieur et inférieur.

Conclusion : L'ETO a permis de confirmer la position correcte de la canule auriculaire gauche et de surveiller l'hémodynamique pendant le PAFG.

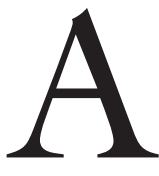
NESTHETIC management of patients requiring repair of the descending aorta is challenging, secondary to the high frequency of coexisting disease and complexity of intraoperative surgical management. Major anesthetic considerations include the management of preexisting medical problems, spinal cord and distal organ protection during aortic cross clamping, single lung ventilation, bleeding, coagulopathy, and postoperative pain management.

Type II Crawford thoracoabdominal aneurysm (TAA) involves most of the descending and abdominal aorta. ${ }^{1}$ Surgical repair can involve the use of an extra-corporeal circuit. In our institution, left atriofemoral bypass (LAFB) technique has been used in 73 patients since October 1997.

Figure 1 shows the components of the LAFB circuit. Details of LAFB, anesthesia, transfusion practice and cooling technique for renal preservation in TAA surgery have been reported elsewhere. ${ }^{2,3}$

Cardiac output is directly affected by the left ventricle end diastolic volume (LVEDV). Estimation of

From the Department of Anesthesia, McMaster University, Hamilton Health Sciences, Hamilton, Ontario, Canada. Address correspondence to: Dr. Ashraf Fayad, Department of Anesthesia, McMaster University, Hamilton Health Sciences, 1200 Main

Street West, Hamilton, Ontario L8N 3Z5, Canada. Phone: 905-521-2100; Fax: 905-523-1224; E-mail: fayad@attcanada.ca Accepted for publication April 8, 2002.

Revision accepted August 9, 2002. 
left ventricular volume using transesophageal echocardiography (TEE) can be obtained either from measurements of cavity area or from volumetric models. ${ }^{4}$ End diastolic cross-sectional left ventricle area, typically in the short-axis view at the midpapillary level, is commonly viewed as a surrogate of volume status. 5,6

In this report we describe the utilization of TEE to confirm position of the left atrial cannula in LAFB. In addition, monitoring of the left ventricular volume assisted hemodynamic management and adjustment of the LAFB pump flow rate.

\section{Case report}

A 57 -yr-old female $(54 \mathrm{~kg})$ presented for type II TAA repair. Her main complaint was persistent backache and dysphagia. Past medical history revealed hypertension, hyperlipidemia and peripheral vascular disease. Current medications included acetyl-salicylic acid and ramipril. Physical examination and laboratory tests were normal except for mild chronic obstructive lung disease. Prior to induction, cerebral spinal fluid drainage was achieved through a lumbar catheter inserted at the L3-4 interspace and a thoracic epidural was inserted at the T8-9 level. Invasive monitoring included both radial and right femoral arterial lines, and a pulmonary artery catheter. Following the induction of general anesthesia with propofol, sufentanil, midazolam and pancuronium, the trachea was intubated with an oral cuffed endotracheal tube (size 7.5) and a left bronchial blocker was inserted. Correct positions of the tube and of the blocker were confirmed using fibreoptic bronchoscopy.

Surgery was carried out with the patient in the right decubitus position. Surgical exposure was achieved through a posterolateral thoracoabdominal incision and excision of the fifth rib. The left atrium was cannulated through the left upper pulmonary vein using a 26-Fr aortic cannula. The left femoral artery was exposed and cannulated with a $24-\mathrm{Fr}$ femoral cannula. The circuit was completed using an electromagnetic centrifugal pump. (Biomedicus ${ }^{\circledR}$ BP-80 electromagnetic centrifugal pump with heat exchanger and a Medtronic Carmeda ${ }^{\circledR}$-coated 3/8" diameter tubing pack; Medtronic Inc., USA).

With initiation of LAFB, difficulty with the pump flow was encountered. The pump was stopped and the circuit was examined for its integrity. A request for TEE was made to verify the position of the left atrial cannula and assist with hemodynamic management.

Echocardiographic images of the left upper pulmonary vein and the left atrium were obtained. The cannula was visualized and noted to be lying against the left atrial wall, and during atrial contractions, com-

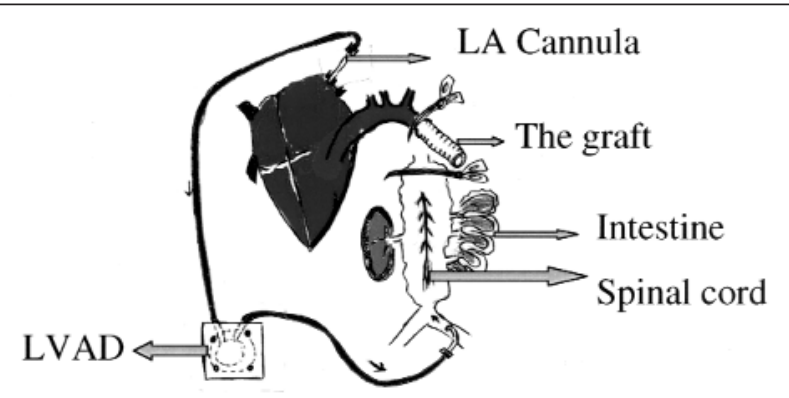

FIGURE I Venous cannula through the upper pulmonary vein, connected to the left ventricle assistance device (LVAD), left ventricle (LV), right ventricle (RV), left atrium (LA)

\section{LA Cannula}

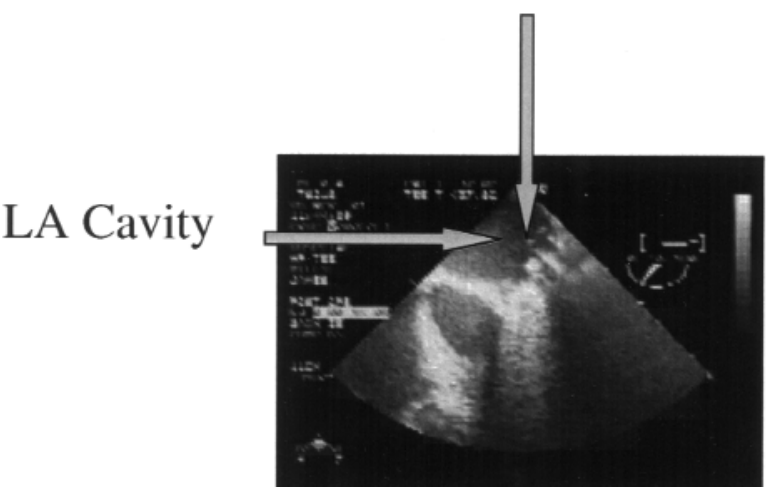

FIGURE 2 The cannula is seen in the left atrium (LA).

plete occlusion of the cannula occurred. The cannula's position was adjusted, and its correct position confirmed by TEE (Figure 2). The LAFB was re-initiated and the TEE image (colour flow Doppler) showed free flow through the cannula. Four video clips marked as initially, trying to advance, advanced and flow may be consulted at cja-jca.org.

During the remainder of the case, TEE was used to monitor left atrial and left ventricular cavity size during different stages of the procedure. Initially, LAFB flow rate was $500 \mathrm{~mL} \cdot \mathrm{min}^{-1}$. TEE images, however, suggested underfilling of the left ventricle. Albumin $5 \% 500 \mathrm{~mL}$ and 21 of Ringer's lactate were administered. Mean arterial pressure was maintained between 
65-75 mmHg. During aortic cross clamping, LAFB flow was increased to over $3 \mathrm{~L} \cdot \mathrm{min}^{-1}$, but subsequently adjusted to between $2.5-3.5 \mathrm{~L} \cdot \mathrm{min}^{-1}$, based on the TEE visualization of left ventricular cavity size.

\section{Discussion}

In this patient, TEE played a key role in confirming the correct position of the left atrial cannula and in monitoring left ventricular volume status.

TAA surgery carries high mortality and morbidity rates but, over the last ten years, the mortality rate has decreased to about 10\%.7, A The use of LAFB has been reported to decrease the incidence of neurologic deficits and renal failure. ${ }^{8,9, \mathrm{~B}}$

TAA repair can be performed by clamping the proximal aorta and suturing expeditiously. This approach relies on surgical speed to limit ischemia. Another approach utilizes the shunting of blood through a conduit inserted between proximal and distal aorta in an attempt to maintain spinal cord, renal, and lower extremity perfusion. A third approach utilizes extracorporeal circulation. This involves clamping the aorta and circulating blood to the lower body via femoral-femoral bypass or LAFB. ${ }^{10,11}$

In this patient, the LAFB technique was used. Flow rates generated to the lower part of the body during LAFB are dependent upon left atial volume, cardiac output and distal vascular resistance. Native cardiac output maintains perfusion to the upper body. The LAFB flow rate determines the amount of blood diverted and the left atrial volume or pressure may no longer reflect the LVEDV. Consequently, pulmonary arterial occlusion pressure may no longer reflect LVEDV. If the LAFB flow rate is high and significant volume is diverted from the left atrium, LVEDV may be compromised, as may perfusion to the upper body. During surgery, significant blood loss and third space fluid shifts also occur. The need to distinguish hypovolemia from a LAFB-induced LV underfilling as the cause for upper body hypotension becomes a clinical challenge. Intraoperative TEE is unique in its ability to provide continuous monitoring of left ventricular function and filling. In fact TEE currently is the only monitor capable of monitoring the size of the left ventricular cavity (LVEDV) in this situation.

\footnotetext{
A Cina CS, Lagana A, Jones DK, Irvine KPA, Clase CM. A meta-analysis of surgical strategies for repair of thoracoabdominal aortic aneurysm. Canadian Society for Vascular Surgery Annual Meeting, Ottawa, Ontario, October 19-21, 2001.

B Cina CS, Lagana A, Bruin G, et al. Thoracoabdominal aortic aneurysm repair: a prospective cohort study of 121 cases. Canadian Society for Vascular Surgery Annual Meeting, Banff, Alberta, September 29-30, 2000.
}

In summary, TEE was used to confirm correct positioning of the left atrial cannula. The hazards of cannula malposition include poor flow rates to the distal organs during TAA repair, and left atrium trauma or rupture. TEE was useful to monitor the left ventricular volume and to adjust the LAFB flow rate. In this case, TEE proved to be a useful adjunct for the management of LAFB during TAA repair.

\section{References}

1 Money SR, Hollier LH. The Management of thoracoabdominal aneurysms: advances in surgery. 1994; 27 : 285-7.

2 Cina CS, Irvine KPA, Jones DK. A modified technique of atriofemoral bypass for visceral and distal aortic perfusion in thoracoabdominal aortic surgery. Ann Vasc Surg 1999; 13: 560-5.

3 Cina CS, Bruin G. Acute normovolemic hemodilution $(\mathrm{ANH})$ in surgery of the thoraco-abdominal aorta. A cohort study to evaluate coagulation parameters and blood products utilization. J Cardiovasc Surg (Torino) 1999; 40: 37-43.

4 Huemer G, Wohlrab C, Greher M. Transesophageal echocardiography and volume management in the intensive care unit. Anaesthesia 1998; 53(Suppl 2): 8-10.

5 Clements FM, Harpole DH, Quill T, Jones RH, $M c$ Cann RL. Estimation of left ventricular volume and ejection fraction by two-dimensional transesophageal echocardiography: comparison of short axis imaging and simultaneous radionuclide angiography. $\mathrm{Br} \mathrm{J}$ Anaesth 1990; 64: 331-6.

6 Troianos CA, Porembka DT. Assessment of left ventricular function and hemodynamics with transesophageal echocardiography. Crit Care Clin 1996; 12: 253-72.

7 Safi HJ. Role of the BioMedicus pump and distal aortic perfusion in thoracoabdominal aortic aneurysm repair. Artificial Organs 1996; 20: 694-9.

8 O'Connor CJ, Rothenberg DM. Anesthetic considerations for descending thoracic aortic surgery: part I. J Cardiothorac Vasc Anesth 1995; 9: 581-8.

9 O'Connor CJ, Rothenberg DM. Anesthetic considerations for descending thoracic aortic surgery: part II. J Cardiothorac Vasc Anesth 1995; 9: 734-47.

10 Coselli JS. Thoracoabdominal aortic aneurysms: experience with 372 patients. J Card Surg 1994; 9: 638-47.

11 Fehrenbacher JW, McCready RA, Hormuth DA, et al. One-stage segmental resection of extensive thoracoabdominal aneurysms with left-sided heart bypass. J Vasc Surg 1993; 18: 366-71. 